\title{
An Economic Model of Bystanders' Behaviour
}

\author{
Yuriko Isada', Nobuko Igaki' ${ }^{1}$ Aiko Shibata ${ }^{2}$ \\ ${ }^{1}$ School of Policy Studies, Kwansei Gakuin University, Hyogo, Japan \\ ${ }^{2}$ Board of Trustee, International Christian University, Tokyo, Japan \\ Email: yuriko@kwansei.ac.jp
}

Received 2 December 2015; accepted 5 January 2016; published 12 January 2016

\begin{abstract}
In this paper, we deal with a classroom where bullying is occurring and assume that if a number of bystanders who tattle bullying exceeds a threshold, then bullying is stopped. Bystanders' behaviour is formulated as a non-cooperative game, with negative externality, retaliation cost and reporting cost. Our research shows that to stop bullying, it is useful to raise the cost of negative externality and to reduce the retaliation cost and or the reporting cost. Additionally, it is shown that small class is effective in preventing bullying.
\end{abstract}

\section{Keywords}

Bullying, Non-Corporative Game, Small Class, Free Rider

\section{Introduction}

Bullying is one of many serious problems in the field of education. The number of recognized incidents of bullying in 2013 was 185,860 according to the results of survey by the Ministry of Education, Culture, Sports, Science and Technology (MEXT) in Japan [1]. In 2013, compared to 2012, the total number of recognized incidents of bullying remained almost unchanged, however, bullying incidents increased at elementary schools. Furthermore, there were 240 student suicides in 2013 compared to 196 students in 2012, 3.8\% of those had been caused by bullying compared 3.1\% in 2012. These facts suggest that the damage caused by bullying had been aggravated and bullying was recognized as crime recently. Magata [2] indicated that most incidents of bullying are criminal acts and illustrated the types of bullying that are criminal activities. Morita [3] emphasised the importance of by standers' behaviour.

In this paper, we consider both the retaliation cost and the reporting cost and focus on the bystander's behaviour. he retaliation cost was dealt in Shibata et al. [4] and the reporting cost was dealt in Isada et al. [5]. Actually, our model in this paper is a generalised model of both models [4] [5].

This paper comprises several sections in which different aspects of the bystander's behaviour around bullying are discussed. Section 2 describes our model in detail. The Nash equilibrium within the model is analysed in section 3. Section 4 discusses numerical experiments with changes to class size and the impact of these changes on bystander's behaviour. Our conclusions are presented in Section 5. 


\section{An Economic Model of by Standers' Behaviour in Bullying}

There are three kinds of students in this situation; the bully, the bullied child and by standers. In this paper we only focus on bystanders' behaviour in a classroom where there is bullying. Suppose that that is $n$ by standers in the class each bystander can take behaviour R, where a student reports bullying to a teacher, or behaviour S, where a student does not report the bullying. Bullying is resolved when more than $t$ students report the bullying.All students are initially granted a utility level $w$. When bullying occurs, students incur a negative externality (disutility) $b$. The retaliation cost $c$ is incurred for students who select behaviour R, only when the bullying could not be stopped despite reporting it. The reporting cost $e$ is constantly incurred for student who selects behaviour R, regardless of whether bullying is stopped or not.

Then, a non-cooperative n-person game model [6] [7] is formulated shown in Table 1. Each value shows the student's gain in each case, where $\mathrm{X}$ denotes the number of reporters other than himself of herself.

\section{Nash Equilibrium in the Economic Model}

Each bystander play this game according to Table 1. Suppose each bystander has the same probability of reporting, $q$. When bystanders other than oneself select behaviour R with a probability $q$, the probability of case 1 , 2 and 3 are $p_{1}(q), p_{2}(q), p_{3}(q)$ respectively as shown below:

$$
\begin{gathered}
p_{1}(q)=p_{1}(q, n, t)=\sum_{i=0}^{t-2}{ }_{n-1} C_{i} q^{i}(1-q)^{n-1-i} \\
p_{2}(q)=p_{2}(q, n, t)={ }_{n-1} C_{t-1} q^{t-1}(1-q)^{n-t} \\
p_{3}(q)=p_{3}(q, n, t)=\sum_{i=t}^{n-1}{ }_{n-1} C_{i} q^{i}(1-q)^{n-1-i} \\
p_{1}(q)+p_{2}(q)+p_{3}(q)=1 .
\end{gathered}
$$

The Expected utility of $E_{R}(q)$ when a bystander selecting behaviour R, and the Expected utility of $E_{S}(q)$ when a bystander selecting behaviour $\mathrm{S}$ are expressed with the following equations.

$$
\begin{aligned}
E_{R}(q)= & p_{1}(q)(w-b-c-e)+p_{2}(q)(w-e)+p_{3}(q)(w-e) \\
= & w-e-(b+c) \cdot p_{1}(q) \\
E_{S}(q) & =p_{1}(q)(w-b)+p_{2}(q)(w-b)+p_{3}(q) w \\
& =w-b \cdot\left(p_{1}(q)+p_{2}(q)\right)
\end{aligned}
$$

$q=1$ is the state when all bystanders select behaviour R and $q=0$ is the state when all bystanders select behaviour S. When $E_{R}(q)=E_{S}(q)$, the result is as $e / b=p_{2}(q)-c / b p_{1}(q)$. Let's denote that $v(q)=p_{2}(q)-c / b p_{1}(q)$.

As we saw in Figure 1, from $v(0)=-c / b$ and $v(1)=0$, there are two values of $q$ which hold $E_{s}(q)=E_{R}(q)$ when $v\left(q_{0}\right)>e / b$ and $e / b>0$ are satisfied, where, $q_{0}$ is vertex of $y=v(q)$. Conversely, if $v(q)<0$ and $e / b \leq 0, y=v(q)$ and $y=e / b$ have only one intersection. When $e=0$, it is shown in Shibata et al. [4]. If $e / b<0$, either of $b$ and $e$ must be negative value. This indicates that when bullying occurs students obtain utility or they get some benefit from reporting the bully. Consequently, we assumed that both of $b$ and $e$ are positive values because such a situation is not demonstrated appropriately by the bystander's behaviour.

Table 1. Changes to student's gain by the number of reporters when selecting either behaviour R or S.

\begin{tabular}{cccc}
\hline & Case $1(\mathrm{X}<=\mathrm{t}-2)$ & Case $2(\mathrm{X}=\mathrm{t}-1)$ & Case 3 $(\mathrm{X}>=\mathrm{t})$ \\
\hline Behaviour R & w-b-c-e & w-e & w-e \\
Behaviour S & w-b & w-b & w \\
\hline
\end{tabular}




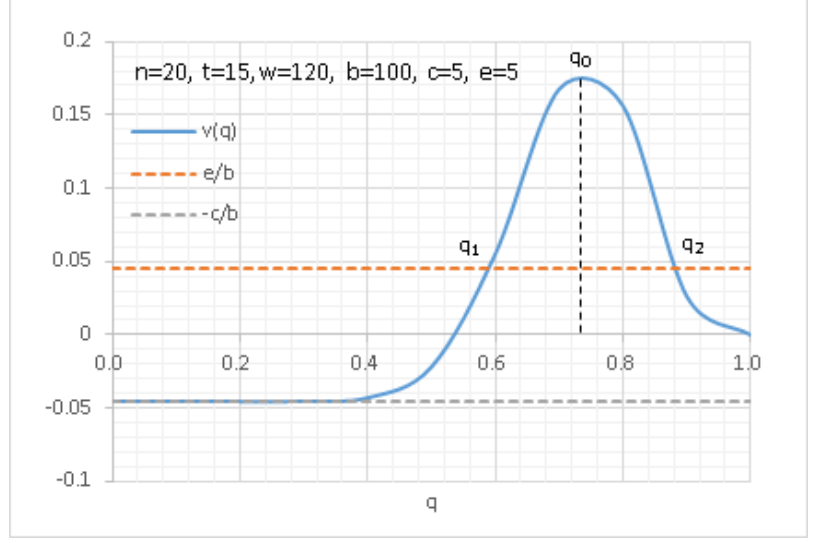

Figure 1. Two intersections of $y=v(q)$ and $y=e / b$.

Let them denote $q_{1}=q_{1}(n, t), \quad q_{2}=q_{2}(n, t)\left(0<q_{1}<q_{0}<q_{2}<1\right)$.

Figure 2 shows the relationship between expected utilities $E_{R}(q)$ and $E_{S}(q)$ and for by stander behaviour $\mathrm{R}$ and $\mathrm{S}$ where $v\left(q_{0}\right)>e / b$ and $e / b>0$.

As we saw in Figure 2, $E_{R}(q)$ and $E_{S}(q)$ always have two intersections for the range $q_{2}<q$, $E_{s}(q)>E_{R}(q)$ occurs. This indicates that a free rider phenomenon occurs where many other students report bullying, but the student in question decides it is better not to report. When $e=0, y=v(q)$ and $y=e / b$ have only one intersection. This case shows a situation where a free rider phenomenon is disappeared. Based on the above, we can make the following proposition.

Proposition 1

1) A pure strategy Nash equilibrium always exists in which no student reports bullying.

2) If $v\left(q_{0}\right)>e / b$ is satisfied, there are two intersection $q_{1}$ and $q_{2}$, then, we have two mixed strategies Nash equilibrium exists.

3) When $v\left(q_{0}\right)>e / b$, a range $\left[q_{1}, q_{2}\right]$ exists for $q$ where $E_{s}(q)<E_{R}(q)$. Conversely, when $v\left(q_{0}\right) \leq e / b$, $E_{S}(q) \geq E_{R}(q)$ is always true.

Figure 3 is a graph of $y=v(q)$ and $y=e / b$ where the number of $c$ is changing and the number of $b$ and $e$ are kept constant. Figure 4 is a graph of $y=v(q)$ and $y=e / b$ where the number of $e$ is changing and the number of band care kept constant.

When examining Figure 1 and Figure 4, we see that we can expand the range $\left[q_{1}, q_{2}\right]$ of $q$ by reducing $e / b$, since $q_{1}$ is decreased and $q_{2}$ is increased. As we saw in Figure 3 the range $\left[q_{1}, q_{2}\right]$ of $q$ is expanded by reducing $c$. Based on the above, we can make the following propositions.

Proposition 2

1) A decrease in $e / b$ due to an increase in $b$ or a decrease in e causes a decrease in $q_{1}$ and an increase in $q_{2}$.

2) A decrease in $c$ causes a decrease in $q_{1}$ and an increase in $q_{2}$.

\section{The Impact of Changing the Class Size}

Let us examine changes in $y=v(q)$ and $y=e / b$ that occur at the two intersections with $q_{1}$ and $q_{2}$ when changing only $n$, the number of bystanders in the class, while the ratio of threshold to the number of bystanders is kept constant at $t / n$. Figure 5 is a graph of $v(q)$ where the number of $n$ is changing. As the value of $n$ becomes smaller, $q_{1}$ becomes smaller and $q_{2}$ becomes larger. Thus, the range $\left[q_{1}, q_{2}\right]$ of $q$ expands.

Figure 6 is a graph that shows the value of $q_{1}$ and $q_{2}$ for six cases, $(n, t)=(20,10),(40,20) \ldots$, where the ratio of $t / n$ keeps a constant $1 / 2$.As $n$ becomes smaller, $q_{1}$ becomes smaller and $q_{2}$ becomes larger. Again, the range $\left[q_{1}, q_{2}\right]$ of $q$ expands. On the other hand, as class size $n$ becomes larger, $q_{1}$ becomes larger and $q_{2}$ becomes smaller. The values of the upper limit $q_{2}$ decrease, and the lower limit $q_{1}$ increase, and the range $\left[q_{1}, q_{2}\right.$ ] of $q$ shrinks. It is shown in Shibata et al. [4] that when $e=0$, that is $e / b=0, q_{1}=q_{1}(n)$ is monotonically decreasing for $n$. 


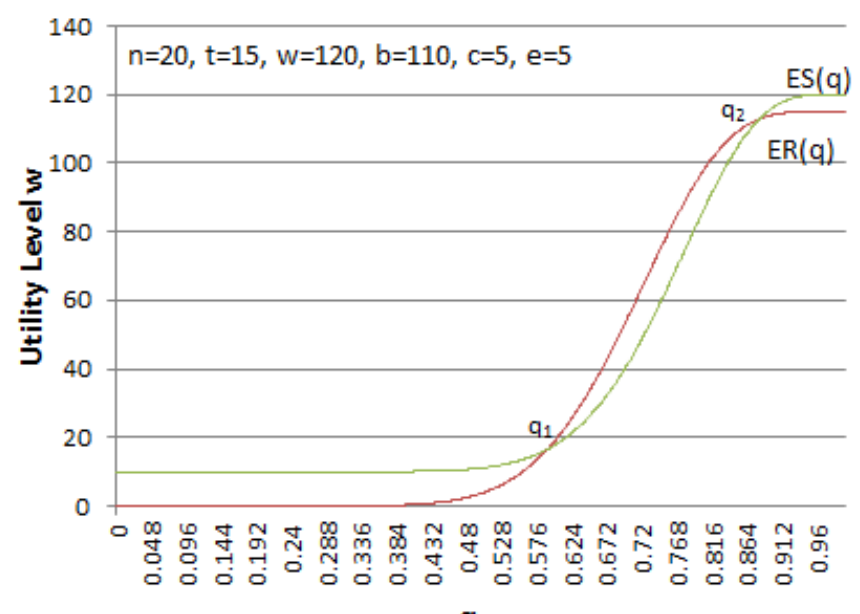

Figure 2. Expected utility when there are two intersections of $y=v(q)$ and $y=e / b$.

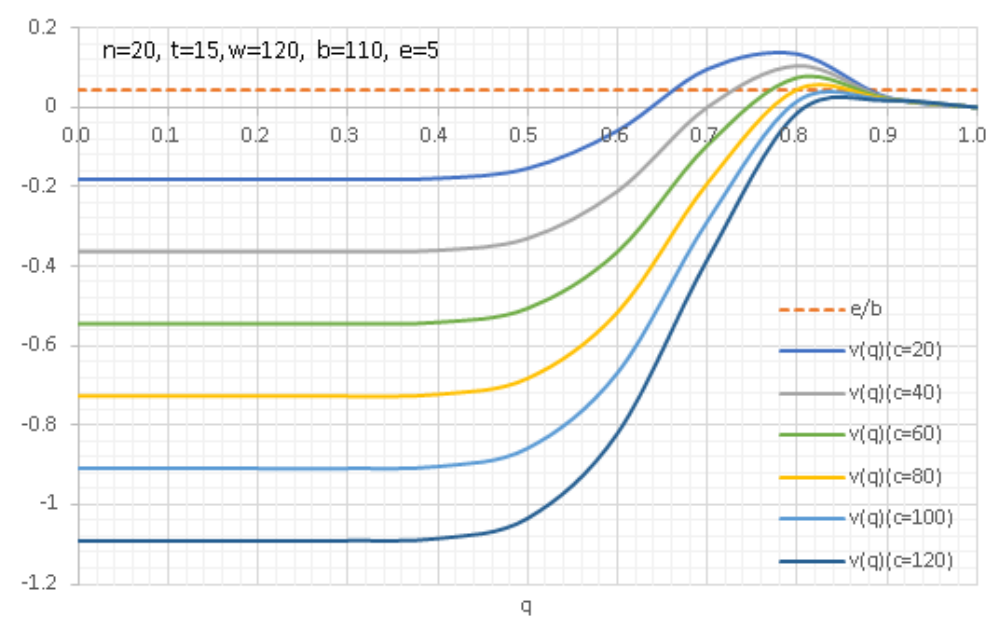

Figure 3. A graph of $v(q)$ when increasing $c$ while $b$ and eare kept constant.

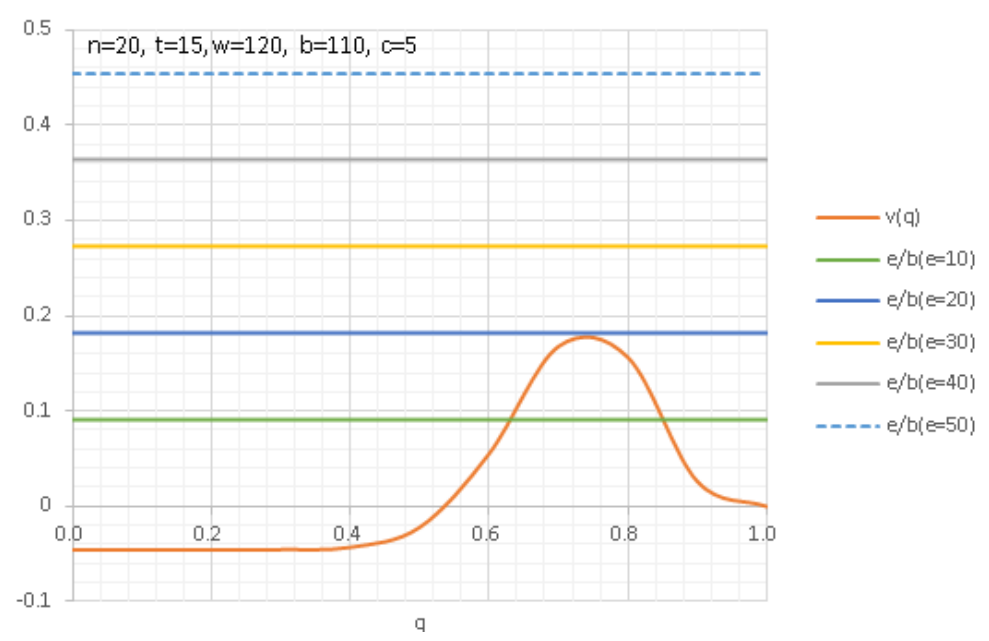

Figure 4. A graph of $v(q)$ when increasing $e$ while $b$ and $c$ are kept constant. 


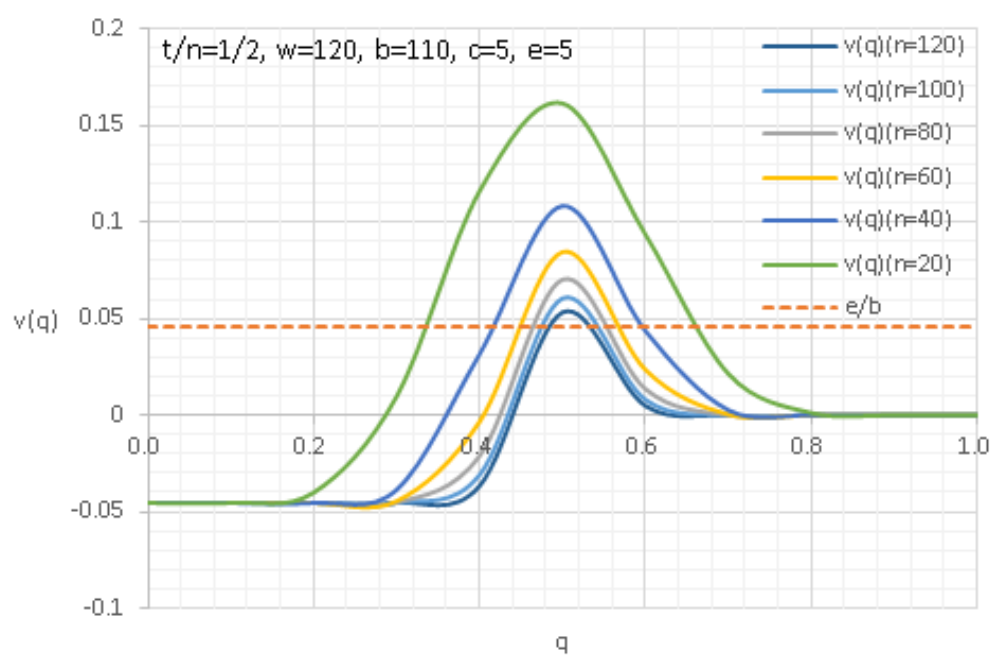

Figure 5. A graph of $v(q)$ when increasing $n$ and $t$ while maintaining $t / n$.

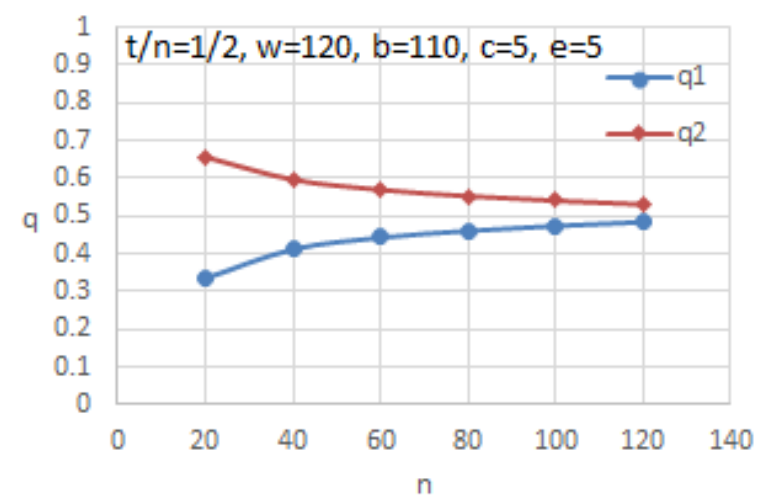

Figure 6. A graph of $q_{1}, q_{1}$ when increasing $n$ and $t$ while maintaining $t / n$.

\section{Conclusion}

In this paper we modelled the bystanders' behaviour with bullying in a non-cooperative n-player game. The three costs are the cost of negative externality, the retaliation cost and the reporting cost, were considered in this model. If the probability of reporting $q$ is larger than the lower limit $q_{1}$, it may increase the chance that bullying is resolved. If the probability of reporting $q$ is larger than the upper limit $q_{2}$, there is a possibility that the free rider phenomenon may arise. This shows that in order to expand the range $\left[q_{1}, q_{2}\right]$ of $q$, it is useful to raise the negative externality $b$ associated with continued bullying and to reduce the retaliation cost $c$ or the reporting cost $e$ of reporting on bullies. We showed by making $t / n$, the ratio of a threshold number of reporters to the number of bystanders, constant and decreasing nit becomes possible to decrease the lower limit $q_{1}$ and to increase the upper limit $q_{2}$ of the probability of reporting bullying. This shows that if class sizes are smaller, it may increase the chance that bullying is stopped because the range $\left[q_{1}, q_{2}\right]$ of $q$ is expanded.

\section{Acknowledgements}

This work was supported by JSPS KAKENHI Grant Numbers 25350468.

\section{References}

[1] MEXT (2014) Results of the 2013 Survey of Problem Behaviour of Students and Various Issues with Instructing Students. http://www.mext.go.jp/b_menu/shingi/chukyo/chukyo3/gijiroku/_icsFiles/afieldfile/2014/12/18/1354117_5.pdf

[2] Magata O. (2013) Ijime to hanzai. [Bullying and Crime] Chuo. 
http://www.yomiuri.co.jp/adv/chuo/opinion/20140303.htm

[3] Morita, Y. (2010) Ijime to ha nanika [What Is Bullying].Chuko Shinsho, Tokyo.

[4] Shibata, A., Mori, T., Okamura, M. and Soyama, N. (2008) An Economic Analysis of Apathetic Behavior: Theory and Experiment. The Journal of Socio-Economics, 37, 90-107. http://dx.doi.org/10.1016/j.socec.2006.12.026

[5] Isada, Y., Igaki, N. and Shibata, A. (2015) A Game-Theoretic Model for Bystanders’ Behaviour in Classes with Bullying. Open Journal of Social Sciences, 3, 97-102. http://dx.doi.org/10.4236/jss.2015.39015

[6] Nash, J. (1950) Equilibrium Points in n-Person Games. Proceedings of the National Academy of Sciences of the United States of America, 36, 48-49. http://dx.doi.org/10.1073/pnas.36.1.48

[7] Nash, J. (1951) Non-Cooperative Games. Annals of Mathematics Second Series, 54, 286-295. http://dx.doi.org/10.2307/1969529 